\title{
Trastornos del lenguaje en alumnos con TEA
}

Enviado: 22 de enero de 2021 / Aceptado: 3 de marzo de 2021 / Publicado: 12 de julio de 2021

MARÍA MARZO-CORDÓN

Estudios de Psicología y Ciencias de la Educación, Universitat Oberta de Catalunya, España mmarzocord@uoc.edu

MÓNICA BELDA-TORRIJOS

Estudios de Psicología y Ciencias de la Educación, Universitat Oberta de Catalunya, España mbeldat@uoc.edu

\section{RESUMEN}

El objetivo de esta investigación es identificar los diferentes problemas del lenguaje que se derivan del Trastorno del Espectro Autista, así como los rasgos identificativos más característicos de cada uno de ellos, atendiendo especialmente a la edad a la que se presentan, así como evaluar la efectividad de las diferentes estrategias de intervención utilizadas en cada caso.

Este trabajo de investigación se ha realizado con 32 participantes, contrastando la información recibida de casos reales intervenidos por docentes y especialistas en el ámbito de la comunidad foral de Navarra. Se ha podido determinar la efectividad de las distintas intervenciones realizadas en los casos, que fueron detectados en diferentes edades, especialmente la efectividad de las intervenciones tempranas, que han obtenido unos resultados muy superiores en el posterior desarrollo de las habilidades del lenguaje de los niños intervenidos.

\section{ABSTRACT}

Language Disorders in Pupils with Intellectual Disabilities

The aim of this research is to identify the different language problems derived from Autism Spectrum Disorder, as well as the most characteristic identifying features of each one of them, paying special attention to the age at which they occur, and to evaluate the effectiveness of the different intervention strategies used in each case.

This research work has been carried out with 32 participants, contrasting the information received from real cases intervened by teachers and specialists in the Autonomous Community of Navarre. It has been possible to determine the effectiveness of the different interventions carried out in the cases, which were detected at different ages, especially the effectiveness of the early interventions, which have obtai- 
Los datos obtenidos tras un análisis técnico realizado en el software de análisis matemático numbers han permitido determinar la efectividad de la intervención temprana poniendo de manifiesto la relevancia de la inmediatez de la detección e intervención y el nivel de efectividad de diversos métodos propuestos en numerosos estudios de referencia.

Palabras Clave: autismo, lenguaje, comunicación, intervención, competencias. ned much better results in the subsequent development of the language skills of the children involved.

The data obtained after a technical analysis carried out in the mathematical analysis software numbers have allowed us to determine the effectiveness of early intervention, highlighting the relevance of the immediacy of detection and intervention and the level of effectiveness of various methods proposed in numerous reference studies.

Keywords: autism, language, communication, intervention, competences.

\section{INTRODUCCIÓN}

El Trastorno del Espectro Autista (TEA) es un trastorno que causa alteraciones en la interacción social, y con esta en la comunicación y el lenguaje, tanto es así que el principal motivo de preocupación entre los padres es la ausencia del inicio del lenguaje cuando el niño ya debería haber iniciado esta etapa.

En la actualidad existen una serie de trastornos del lenguaje asociados a los niños autistas como el mutismo selectivo o el síndrome fonológico-sintáctico, que es el más común no solo en niño que presentan el trastorno del lenguaje autista, también en los que no lo presentan, así como el trastorno semántico-pragmático del lenguaje, que es el más característico y el más relacionado con las alteraciones en la interacción social causante de la dificultad para iniciar una conversación o mantener los turnos de palabra, entre otros trastornos tales como el mutismo selectivo. En cualquier caso, el TEA es un trastorno del desarrollo que se inicia de manera precoz, y es un hecho contrastado que el tratamiento temprano de los trastornos del lenguaje de los niños con TEA se traduce en unos mejores resultados de la intervención educativa, lo que permite obtener evoluciones más positivas en su tratamiento.

En base a esta premisa, la detección del TEA y aquellos trastornos del lenguaje asociados a él debe ser por lo tanto lo más temprana posible. 
Los trastornos del lenguaje de los niños autistas y no autistas parecen no diferir mucho, al menos de forma general, como se ha mencionado el síndrome fonológico-sintáctico es el común en ambos casos, a pesar de lo cual existen ciertas diferencias que podemos advertir y que deben ser investigadas para su detección y con ésta el inicio de la intervención.

Existe un abrumador volumen de información al respecto, resultando en múltiples teorías a menudo contradictorias y opiniones profesionales adversas que muchas veces confunden a padres y educadores. Este es uno de los principales problemas con los que un educador entra en contacto al comenzar su andadura profesional, por lo que una investigación a cerca de la detección de este tipo de trastornos y su intervención podría proporcionarme la información de primera mano que podré aplicar en mi profesión

El problema fundamental de los trastornos del lenguaje radica en las nefastas consecuencias que acarrean las dificultades derivadas de los problemas en el desarrollo de las habilidades comunicativas en el ámbito social y fundamentalmente en el de la educación. Podemos asegurar sin temor a equivocarnos que la base de la educación es la comunicación, y la ausencia de una adecuada comunicación con sus padres y educadores es un impedimento para el correcto desarrollo del niño en casi todos los aspectos de su educación incluyendo el desempeño de la labor de padres y educadores, pues la educación es una parte integral de cualquier proceso de enseñanza aprendizaje que pretenda ser funcional.

Ayudar a niños con TEA a lograr una adecuada comunicación con su familia y educadores es de vital importancia, priorizar la intervención temprana en los trastornos del lenguaje asociados al TEA es una parte fundamental para garantizar una educación de calidad.

En base a lo publicado en numerosos artículos (Fortea-Sevilla (2015), Martos y Ayuda (2002), Artigas (1999) todos los niños, en ausencia de problemas biológicos que se lo impidan, desarrollan el lenguaje oral en mayor o menor medida durante su desarrollo, sin embargo, el retraso en la adquisición del lenguaje parece estar asociado en numerosas ocasiones al TEA.

Conocemos bien algunas características genéricas que acompañan a este trastorno como la dificultad social para manejar las relaciones sociales, incluso el retraso en la adquisición del lenguaje, pero a todas luces debemos abordar estos problemas de forma individual para cada niño, atendiendo a su propia experiencia vital y capacidades. Así pues, un aspecto fundamental a tener en cuenta en nuestra investigación es que cada niño es diferente y único y así debe ser entendido para un desarrollo efectivo de cualquier metodología de intervención.

La convención entre los profesionales es que la detección y tratamiento deben darse de manera temprana e individualizada, no obstante, no existe una convergencia sobre la metodología 
más eficaz a la hora del tratamiento de estos trastornos. Por esta razón para estar completo este estudio no solo debe comprender la detección sino contemplar además los diferentes métodos de intervención, asumiendo que ésta debe prestarse de la forma temprana posible. De esta manera, aunque existe convergencia en la conveniencia de realizar la intervención para el tratamiento de estos trastornos de forma precoz y no a la hora de seleccionar la metodología más adecuada, podemos advertir en general sí hay estudios pediátricos y pedagógicos que pueden darnos algunas pistas para sentar las bases del problema con datos contrastados y verificables.

En septiembre de 2005, 54 equipos de pediatría participaron en un programa de cribado para la detección temprana de trastornos, administrando un cuestionario M-CHAT, hasta 2012 un total de 9.524 niños participaron en dicho programa, un programa ambicioso en el número de participantes (tanto profesionales como niños) y en el tiempo que duró el estudio que se extendió durante 4 años, un $80 \%$ de los equipos participantes evaluaron de gran utilidad los test para la detección y diagnóstico de estos problemas, el 20\% restante los evaluaron de utilidad con algunas reservas. (Programa de detección precoz de trastornos generalizados del desarrollo en las áreas de salud de Salamanca y Zamora.) Existen estudios previos que ponen en valor la importancia de atender estos casos de forma temprana, por ello la identificación prematura de las dificultades comunicativas asociadas a este trastorno es fundamental para desarrollar estrategias efectivas en su tratamiento y la consecución del mejor desarrollo posible de las capacidades del lenguaje y la comunicación en estos niños.

En aras de proveer de una herramienta eficaz para la detección e intervención de estos trastornos el objetivo principal de este estudio es investigar todos aquellos rasgos identificativos propios de los trastornos del lenguaje y la comunicación en niños con TEA y averiguar cuales son los tratamientos más efectivos contrastando resultados de diferentes métodos de intervención aplicados en casos reales. Para ellos necesitaremos acotar la muestra del estudio por medio de la edad, centrándonos en el rango comprendido entre los 0 y 6 años, es decir, primer y segundo ciclo de Educación Infantil.

En esta etapa se desarrolla el lenguaje y se forjan las habilidades comunicativas, por lo que trataremos de llegar a la mayor muestra posible dentro de este rango de edades, tratando de mantener en todo momento un estrecho contacto con familias y educadores involucrados en cada caso real que integra nuestro estudio.

La relación de los trastornos del lenguaje y la comunicación asociados al TEA con los rasgos que los identifican constituye una herramienta muy útil a la hora de su diagnóstico temprano, para de esta manera, realizar una intervención eficaz que permita un correcto 
desarrollo de las capacidades comunicativas. Así, teniendo en cuenta la información de la que disponemos y los objetivos perseguidos en el estudio surgen diversas preguntas que la investigación debe tratar de resolver: ¿Qué trastornos del lenguaje y la comunicación están asociados al TEA y qué métodos son mejores para el tratamiento precoz de estos trastornos?

Conocemos muy bien dos factores determinantes a la hora de realizar la intervención cuando un niño presenta dificultades en el lenguaje y la comunicación relacionadas con este trastorno, la estrategia debe ser temprana y diseñada en función de las necesidades específicas del niño y sus vivencias y situación personal. Una vez seamos capaces de hallar respuesta a estas cuatro preguntas contaremos con el resto de las herramientas que nos permitan ayudar a los niños que presentan esta problemática a desarrollar sus capacidades comunicativas y con ellas garantizar su acceso a la educación, complementando de esta manera la información que ya tenemos.

A modo de breve resumen podemos enumerar las pretensiones del presente estudio de la siguiente manera: identificar aquellos rasgos que delaten la presencia de trastornos del lenguaje en niños con TEA de manera temprana y los métodos de intervención que aporten un mejor resultado para cada uno de ellos mediante el estudio de casos reales en una muestra lo más amplia posible comprendida en el rango de edad de 0 a 6 años, la etapa de Educación Infantil.

\section{MATERIAL Y MÉTODO}

Como en toda investigación educativa perseguimos construir conocimientos relativos a una realidad social que nos aporten respuestas a un problema, en este caso los derivados del trastorno del aspecto autista y relativos al lenguaje y el habla. Para llevar a cabo este estudio se ha optado por realizar una investigación cuantitativa contando para ello con una muestra representativa del colectivo de la educación. La muestra esta formada por docentes de diferentes centros educativos de la comunidad foral de Navarra, en el ámbito de la detección e intervención de los problemas del lenguaje en alumnos con TEA.

Esta investigación se basa en la recopilación de información proporcionada por los participantes mediante preguntas estandarizadas, posteriormente analizada para extraer los conocimientos necesarios que permitan contar con un apoyo para seleccionar la metodología más efectiva en cada caso. Este tipo de investigaciones cuantitativas se orientan a la obtención de explicaciones mediante el análisis de los datos previamente recopilados en forma de estudio precientífico (Galtung, 1966). 
En la recopilación de datos llevada a cabo durante el estudio, se ha recabado información sobre las diferentes propuestas metodológicas empleadas por los docentes en sus intervenciones para tratar con los problemas de lenguaje de los niños con TEA, en las características a partir de las cuales detectaron estos problemas y en las particularidades del contexto de cada caso.

Para obtener un resultado satisfactorio el análisis de los datos debe ser adecuado para revelar su importancia a través de métodos apropiados (Gerardo Meza Cascante, 2002). Para ello descomponemos los datos en unidades de información que estudiamos de forma individual y en relación con la totalidad y nos apoyamos para ello en el análisis de la muestra. Tal como se indica, los datos recopilados se han de descomponer en unidades de información que se estudiarán de manera individual, por ello se debe tomar en consideración las características personales de cada niño y niña para complementar, a través del testimonio de la muestra, la información puramente estadística a fin de comprender mejor la realidad tras los datos.

\section{Participantes}

Para la obtención de los datos a partir de los cuales desarrollar el trabajo de análisis y posterior conclusión de esta investigación, se ha contado con la participación de numerosos docentes de diversos centros de Educación Infantil y Primaria de la comunidad Foral de Navarra. También se ha contado con la información proporcionada por diversos especialistas de PT, AL y Orientación. Para obtener una muestra representativa se ha contado con profesionales tanto del sector público como el privado del mayor número de centros posibles. Al requerirse información precisa de casos reales de intervenciones educativas, se ha optado por no difundir aquella información personal de los niños y niñas que han protagonizado cada caso y de sus familias y que no sea esencial para el desarrollo del estudio para garantizar sus derechos. Para acceder a los participantes se han utilizado los siguientes medios de difusión: teléfono, correo electrónico y aplicaciones de mensajería instantánea, intentando llegar a la mayor cantidad posible de participantes a través de canales especializados.

En primer lugar, se le ha expuesto a cada uno de los docentes el tema del estudio y sus implicaciones, se han enumerado los datos que se van a recabar y el uso que se va a hacer de ellos, así mismo se les ha comunicado que se respetará en todo momento la ley orgánica de protección de datos y la normativa y política de cada centro. Tras aceptar las condiciones de participación y adherirse al estudio se les ha entregado un formulario en dos fases, una sección de preguntas cerradas para la recopilación de datos y una sección abierta para conocer detalles adicionales, esta última parte a pesar de necesitar un proceso más complejo es ne- 
cesaria puesto que la educación requiere de cierta flexibilidad y adaptación personalizada. El número total de docentes que han participado ha sido 36, el número total de centros que han participado ha sido 12, el número total de casos reales registrados ha sido 41 y el número total de casos válidos ha sido 32 .

\section{Instrumentos}

Para la obtención de datos se ha tenido en cuenta muchas de las recomendaciones de la Metodología de la investigación educativa (Bisquerra, 2004), a partir de sus orientaciones se ha optado por realizar la encuesta de forma telemática por su rapidez, por evitarse posibles sesgos del entrevistador y por el mayor acceso a los participantes de diversas zonas de la comunidad, además facilita el análisis cuantitativo posterior.

Se ha diseñado un cuestionario en dos fases; la primera parte ha constado de preguntas cerradas que debían ser respondidas de forma concisa y objetiva, la segunda parte consistía en una sección abierta para recabar detalles específicos de cada intervención. Para entablar y mantener el contacto con los participantes se han utilizado tres medios de comunicación diferentes, el teléfono, el correo electrónico y las aplicaciones de mensajería instantánea, siempre dentro de canales de comunicación cerrados y participados por profesionales de la educación.

En cuanto a los materiales que han posibilitado la realización de este estudio se han utilizado: el Manual de metodología de la investigación educativa, de Rafael Bisquerra Alzina, como material orientativo, ordenadores con conexión a internet, el software de edición de texto MS Word, el de redacción y formato de encuestaras Google Forms y el de procesamiento de datos MS Excel, así como los canales de comunicación anteriormente descritos.

\section{Procedimiento}

Para llevar a cabo esta propuesta de forma satisfactoria, el desarrollo se ha estructurado en tres partes antes de la obtención de resultados: Elaboración del cuestionario Contacto y reclutamiento de los participantes, análisis de la muestra y eflexión en base a los datos obtenidos. Para la elaboración del cuestionario se ha contado con la colaboración del equipo profesional de la UAE (Unidad de Apoyo Educativo) del colegio público de infantil y primaria San Bartolomé (Ribaforada, Navarra). La mencionada unidad está compuesta por dos especialistas de PT, una especialista de AL, personal de orientación y personal de apoyo a minorías. Este cuestionario ha sido diseñado con el objetivo de recabar información sobre las siguientes 
áreas: los problemas del lenguaje derivados del TEA, las características que han posibilitado la detección de dichos trastornos, la edad a la que fueron diagnosticados, las metodologías de intervención empleadas, los resultados obtenidos de dicha intervención, y por último, se ha concedido un espacio abierto para aportar aquellos detalles específicos de cada caso que puedan ser relevantes para el trabajo de investigación.

Se ha cuidado especialmente la selección de las preguntas incluidas puesto que la clave del éxito de nuestra investigación depende directamente de tanto de este factor como de la selección de las técnicas de análisis que se emplearán posteriormente para procesar las respuestas de los participantes. Cada participante ha rellenado un formulario por cada intervención realizada.

La fase de contacto y reclutamiento de los participantes se ha llevado a cabo mediante vía telemática y se ha logrado una participación de 36 profesionales de la educación. Todos los participantes son docentes y especialistas titulados y con experiencia de al menos un año en la educación y se han acreditado como tales a la hora de entregar los cuestionarios.

Para contactar con los participantes se han utilizado distintos canales especializados de interlocución previamente verificados, acudiendo así mismo a los centros de Educación Infantil y Primaria y sindicatos de trabajadores públicos de educación de la comunidad foral de Navarra.

Los participantes han dispuesto de una semana de tiempo para reflexionar sobre cada intervención realizada durante su experiencia laboral y recabar la información y documentación necesaria para contestar al cuestionario de forma precisa. Tras entregar los formularios cumplimentados, los participantes han dispuesto del plazo de una semana para complementar la información proporcionada, tanto por iniciativa propia como alentados por el equipo de investigación cuando ha se ha estimado necesario. En la última fase de la investigación previa a la extracción de los resultados se ha elaborado un análisis de la muestra con la información obtenida:

- Para relacionar los problemas del lenguaje derivados del trastorno del lenguaje autista detectados con las características que posibilitaron su detección y la edad a la que fueron detectados.

- Para relacionar las metodologías empleadas con los resultados obtenidos en cada caso.

Una vez procesados estos datos y a partir de los resultados arrojados por el análisis se obtienen los resultados que persigue la presente investigación educativa, la clasificación de los problemas del lenguaje derivados del TEA según sus características propias y la metodología de intervención adecuada. 


\section{Análisis de la muestra}

El análisis de los datos debe ser amplio y flexible, tal como lo indica Cormack (1971).

La clave para un correcto análisis de la muestra es seleccionar las técnicas en función del objetivo de nuestra investigación. En base a este planteamiento el procedimiento a seguir se ha estructurado de la siguiente manera:

- Verificación: Se ha contrastado la validez y fiabilidad de los datos obtenidos.

- Codificación: Se han traducido a números los datos obtenidos en la fase uno del cuestionario (preguntas cerradas).

- Clasificación: Se han clasificado los datos obtenidos en categorías llamadas variables.

- Procesamiento: Se han registrado el número de casos de cada categoría de variables.

- Presentación: Se presentan los resultados de los anteriores procesos de forma ordenada para su interpretación.

\section{RESULTADOS}

Los resultados obtenidos en la encuesta están orientados a la consecución de los dos principales objetivos de la investigación; En primer lugar verificar la influencia que tiene la detección temprana de los problemas del lenguaje derivados del TEA y su intervención satisfactoria, así como las características que han permitido detectar cada trastorno, en segundo lugar, averiguar que estrategias de intervención están empleando los docentes y profesionales de la educación especial en este tipo de casos y los resultados obtenidos en el desarrollo de las habilidades del lenguaje en los casos en los que se han aplicado.

Tras un primer análisis global de la información recabada, lo primero que nos llama la atención es la cantidad de educadores que no ha recibido ningún tipo de formación específica relativa al TEA, solo un 51\% de los encuestados había recibido formación específica, en su mayoría profesionales de PT y orientación.

Enfrentando este dato con el número de casos y el resultado de mejora (Tabla 1.), obtenemos la relación entre la formación de los educadores y la eficacia de la intervención educativa. De los 9 casos en los que la intervención educativa no logró progresos solo en 3 casos se había recibido formación por parte del profesorado. 
Tabla 1. Relación Formación-Progreso

\begin{tabular}{|c|c|c|c|c|c|}
\hline CASO & FORMACIÓN & MEJORÍA & CASO & FORMACIÓN & MEJORÍA \\
\hline 1 & SI & SI & 17 & NO & NO \\
\hline 2 & SI & SI & 18 & NO & NO \\
\hline 3 & SI & SI & 19 & NO & SI \\
\hline 4 & NO & SI & 20 & SI & SI \\
\hline 5 & SI & NO & 21 & NO & SI \\
\hline 6 & SI & NO & 22 & SI & SI \\
\hline 7 & SI & SI & 23 & NO & SI \\
\hline 8 & SI & SI & 24 & NO & SI \\
\hline 9 & SI & SI & 25 & NO & NO \\
\hline 10 & SI & SI & 26 & NO & NO \\
\hline 11 & NO & NO & 27 & NO & SI \\
\hline 12 & SI & SI & 28 & NO & SI \\
\hline 13 & NO & SI & 29 & SI & $\mathrm{SI}$ \\
\hline 14 & SI & SI & 30 & SI & SI \\
\hline 15 & SI & SI & 31 & SI & NO \\
\hline 16 & NO & NO & 32 & SI & SI \\
\hline
\end{tabular}

Para terminar de determinar la influencia de la formación del profesorado en la detección e intervención de los problemas del lenguaje derivados del TEA, preguntamos a las familias, las cuales no habían recibido ninguna formación al respecto, si habían notado alguna de las alteraciones más frecuentes previas al comienzo de la Educación Infantil.

Un 45,9\% de las familias no había notado ninguna alteración a pesar de que posteriormente su hijo o hija fue diagnosticado de un problema del lenguaje derivado del TEA.

La mayoría de quienes sí notaron alguna alteración fue a cerca de la falta de desarrollo de gestos, salvo para señalar (21,6\%), seguidos de la emisión de palabras carentes de sentido comunicativo $(13,5 \%)$.

Aunque la estadística por su propia condición no puede ser determinante, pone en relieve la importancia de la formación previa en la detección temprana de los trastornos que ocupan en presente estudio.

Los resultados arrojadas en estudios previos, como el realizado en septiembre de 2005 por el programa de detección precoz de trastornos generalizados del desarrollo en las áreas de salud de Salamanca y Zamora, ponen de manifiesto la necesidad de la detección temprana de los 
Figura 1. Alteraciones previas detectadas por la familia

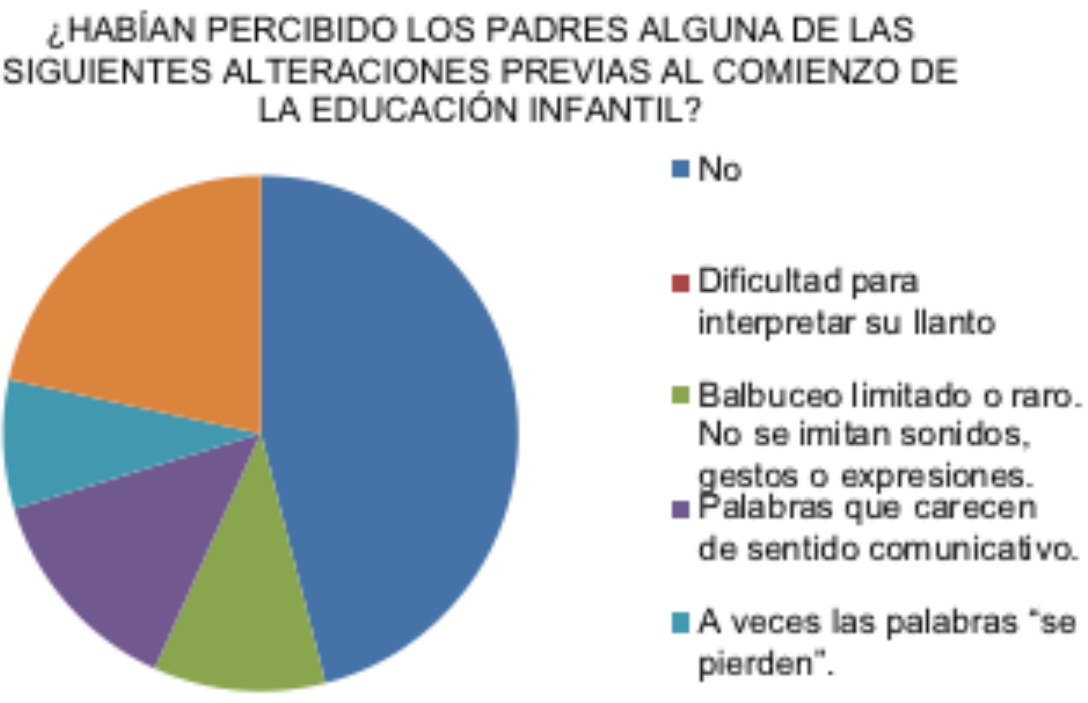

problemas derivados del TEA, incluidos los trastornos del lenguaje relacionados, así, un 80\% de los especialistas consultados manifestaron la utilidad de realizar test de manera temprana.

Tabla 2. Correspondencia edad de detección / evolución

\begin{tabular}{cccc}
\hline EDAD & 1-3 AÑOS & 4-6 AÑOS & 7-9 AÑOS \\
\hline No CASOS DETECTADOS & 7 & 16 & 9 \\
\hline MEJORÍA & 7 & 9 & 7 \\
\hline \% Mejoría/Casos & $100 \%$ & $56,25 \%$ & $77,77 \%$ \\
\hline
\end{tabular}

Un análisis de los datos obtenidos respecto a la edad de detección de los problemas del lenguaje derivados del TEA su evolución (Tabla 2.), revela que la mayoría de los casos intervenidos por los profesionales de la educación que participaron en nuestro estudio fueron detectados entre los 4 y los 6 años de vida del niño. Solo el 21\% de los casos se detectó antes de los 3 años, sin embargo, en la totalidad de los casos detectados en este rango de edad la evolución del desarrollo educativo en lo que concierne al área del lenguaje fue valorada positivamente.

No podemos hacer una afirmación determinante en base a una estadística, pero no cabe duda de que los resultados apuntan a una estrecha relación entre la intervención temprana de estos trastornos y la posterior evolución, siendo más sólido el desarrollo cuanto antes se 
detecten las necesidades especiales del niño y se reorienten las metodologías y estrategias de intervención.

Los trastornos del lenguaje presentes en los niños con TEA pueden detectarse mediante la observación de las alteraciones en el proceso de adquisición del lenguaje.

Durante el estudio se han observado diversas alteraciones siendo las más habituales la Imposibilidad para conversas $(52,8 \%)$ y el uso de Oraciones raras y pobre articulación (30,6\%) (Tabla 3.)

Tabla 3. Alteraciones durante el proceso de adquisición del lenguaje

\begin{tabular}{cccccc}
\hline $\begin{array}{c}\text { Oraciones raras y } \\
\text { pobre articulación }\end{array}$ & $\begin{array}{c}\text { Ecolalia, } \\
\text { problemas } \\
\text { de evocación }\end{array}$ & $\begin{array}{c}\text { Falta de } \\
\text { comprensión } \\
\text { y expresión }\end{array}$ & $\begin{array}{c}\text { Imposibilidad } \\
\text { de conversar }\end{array}$ & $\begin{array}{c}\text { Inversión } \\
\text { pronominal }\end{array}$ & $\begin{array}{c}\text { Preguntas escasas } \\
\text { y repetitivas }\end{array}$ \\
\hline 11 & 8 & 10 & 19 & 1 & 9 \\
\hline
\end{tabular}

La imposibilidad de conversar es con diferencia la alteración que más se detectó durante el estudio, en la mayoría de los casos unida al diagnóstico de Mutismo Selectivo (un total de 8 de los 32 casos estudiados así fueron diagnosticados 5 de ellos en el primer ciclo de Educación Infantil). La ecolalia, Falta de comprensión, uso de oraciones raras y pobremente articuladas y el empleo de preguntas escasas y repetitivas son alteraciones comunes, presentes en entre el 22 y 34\% de los casos estudiado, arrojando la inversión pronominal un resultado casi marginal, inferior a un 3\%. Ha de indicarse que el total de alteraciones detectadas no tiene por que coincidir con el total de casos, pues es común la aparición de múltiples alteraciones en un mismo caso.

El estudio ha incluido el seguimiento de las principales características propias de los distintos tipos de trastornos del lenguaje relativos al TEA. (Tabla 4.)

Tabla 4. Alteraciones durante el proceso de adquisición del lenguaje

\begin{tabular}{cccccc}
\hline $\begin{array}{c}\text { No es capaz de } \\
\text { decodificar el } \\
\text { lenguaje }\end{array}$ & $\begin{array}{c}\text { Utiliza al adulto úni- } \\
\text { camente como medio } \\
\text { para satisfacer necesi- } \\
\text { dades }\end{array}$ & $\begin{array}{c}\text { Lenguaje poco in- } \\
\text { teligible / Déficit } \\
\text { sintáctico }\end{array}$ & $\begin{array}{c}\text { Dificultad para } \\
\text { evocar la pala- } \\
\text { bra adecuada }\end{array}$ & $\begin{array}{c}\text { Mutismo } \\
\text { Selectivo }\end{array}$ & $\begin{array}{c}\text { Disfunciones en la } \\
\text { entonación y ritmo }\end{array}$ \\
\hline 7 & 12 & 11 & 8 & 12 & 7 \\
\hline
\end{tabular}

Una vez más el mutismo selectivo es una característica destacable por su presencia en casi una tercera parte de los casos estudiados, igualmente cuando el niño trata de utilizar al adulto únicamente como medio para satisfacer sus necesidades, generalmente señalando lo que quie- 
re sin emitir palabras. Los resultados del estudio muestran una elevada presencia del déficit sintáctico como característica presente en casi una tercera parte de los casos estudiados.

No en todos los casos han sido detectados específicamente trastornos del lenguaje, siendo tratados de forma más generalizada como TEA (3), TDAH (1) Asperger (1) N/C (4). En la mayoría de los casos estudiados se han diagnosticado específicamente trastornos del lenguaje. (Tabla 5.)

Tabla 5. Diagnósticos Trastornos del lenguaje derivados del TEA

\begin{tabular}{cccccccc}
$\begin{array}{c}\text { Agnosia } \\
\text { Auditiva } \\
\text { Verbal }\end{array}$ & $\begin{array}{c}\text { Síndrome } \\
\text { Fonológico } \\
\text { Sintáctico }\end{array}$ & $\begin{array}{c}\text { Síndrome Léxi- } \\
\text { co Sintáctico }\end{array}$ & $\begin{array}{c}\text { Síndrome } \\
\text { Semántico } \\
\text { Pragmático }\end{array}$ & $\begin{array}{c}\text { Mutismo- } \\
\text { Selectivo }\end{array}$ & $\begin{array}{c}\text { Trastorno de } \\
\text { Prosodia }\end{array}$ & $\begin{array}{c}\text { Trastorno mixto } \\
\text { del Lenguaje }\end{array}$ & Otros \\
\hline 2 & 1 & 7 & 2 & 9 & 1 & 1 & 9 \\
\hline
\end{tabular}

Los mas habituales trastornos diagnosticados son el Síndrome Léxico Sintáctico con un total del $22 \%$ de los casos y el Mutismo Selectivo con un $29 \%$.

Poniendo en relación los trastornos estudiados con las observaciones realizadas por los profesionales de la educación que han participado en el proyecto, las características observada en de cada uno de ellos son:

- Agnosia Auditiva Verbal: El niño se muestra incapaz para decodificar el lenguaje recibido mediante la vía auditiva.

- Síndrome Fonológico Sintáctico: Junto al mutismo selectivo ha sido el trastorno TEL derivado del autismo más observado en el estudio. Muestra un lenguaje poco inteligible por carecer de riqueza semántica y vocalización eficiente.

- Síndrome léxico-sintáctico: Según resultados del estudio en este diagnóstico se muestra dificultad para evocar la palabra adecuada.

- Síndrome semántico-pragmático: Dificultad para comprender al interlocutor, aquí la dificultad radica en que se conjugan los aspectos formales y el uso social y comunicativo del lenguaje.

- Mutismo selectivo: No hacen prácticamente uso del lenguaje, aunque tienen la capacidad para hacerlo.

- Trastorno de prosodia: Dificultad en la entonación y ritmo aplicado al lenguaje.

El uso de estrategias de intervención y criterios metodológicos orientados a salvar estas dificultades es imprescindible para lograr la mejor evolución posible en el desarrollo educativo de los niños con Trastornos del lenguaje relativos al TEA. 
Los datos analizados muestran la frecuencia de uso de diferentes criterios metodológicos y la efectividad registrada, aunque todos los participantes en el estudio afirman haber aplicado una combinación de dos o mas de los siguientes criterios (Tabla 6.)

Tabla 6. Estrategias de intervención educativa y metodologías empleadas

\begin{tabular}{lcc}
\hline \multicolumn{1}{c}{ ESTRATEGIAS } & $\begin{array}{c}\text { FRECUENCIA } \\
\text { DE USO }\end{array}$ & EVOLUCIÓN POSITIVA \\
\hline Tomar los propios intereses del alumno como punto de partida & $61,5 \%$ & $14 / 24$ \\
\hline $\begin{array}{l}\text { Preparación de situaciones que favorecen o desencadenan actos comunicati- } \\
\text { vos }\end{array}$ & $53,8 \%$ & $13 / 21$ \\
\hline $\begin{array}{l}\text { Facilitar los apoyos necesarios para que pueda realizar la actividad y posterio- } \\
\text { rmente retirarlos progresivamente }\end{array}$ & $46,2 \%$ & $11 / 18$ \\
\hline Atención especial a la expresión y comprensión de los estados emocionales & $56,4 \%$ & $15 / 22$ \\
\hline Establecer rutinas y situaciones muy estructuradas que faciliten la anticipa- & $64,1 \%$ & $16 / 25$ \\
\hline ción & & $7 / 12$ \\
\hline Evitar elementos de distracción & $30,8 \%$ & $8 / 18$ \\
\hline Uso de agendas & $46,2 \%$ & $5 / 14$ \\
\hline Aprendizaje colaborativo & $35,9 \%$ & $7 / 16$ \\
\hline Uso de SAAC (sistemas de comunicación aumentativos y alternativos) & $42 \%$ & \\
\hline
\end{tabular}

Las principales estrategias utilizadas durante la intervención educativa por los participantes del estudio han sido:

- Tomar los propios intereses del alumno como punto de partida

- Preparación de situaciones que favorecen o desencadenan actos comunicativos.

- Facilitar los apoyos necesarios para que pueda realizar la actividad y posteriormente retirarlos progresivamente.

- Atención especial a la expresión y comprensión de los estados emocionales.

- Establecer rutinas y situaciones muy estructuradas que faciliten la anticipación.

- Evitar elementos de distracción.

- Uso de agendas.

- Aprendizaje colaborativo.

- Uso de SAAC (sistemas de comunicación aumentativos y alternativos). 
Las estrategias orientadas al establecimiento de rutinas y situaciones estructuradas que facilitan la anticipación son las más comúnmente utilizadas habiéndose implementado en más de la mitad de los casos estudiados $(64,1 \%)$, seguidas por el uso de los propios intereses del alumno como punto de partida para establecer actividades que impliquen el fomento del desarrollo de las habilidades comunicativas y del lenguaje.

La Atención especial a la expresión y comprensión de los estados emocionales es la estrategia cuyos resultados obtienen un mayor número de positivos en cuando a la evolución en el desarrollo educativo satisfactorio según las observaciones de los participantes contribuyendo desarrollo de la adquisición de habilidades comunicativas en el 68\% de los casos. En segundo lugar, con un $64 \%$ de positivos en evolución del desarrollo educativo en el área del lenguaje se encuentra el establecimiento de rutinas y situaciones muy estructuradas que faciliten la anticipación por parte del alumno.

Entre el 58\% y el 61\% de los casos en los que se han aplicado las siguientes estrategias han experimentado una mejoría en el desarrollo del lenguaje: Tomar los propios intereses del alumno como punto de partida, preparación de situaciones que favorecen o desencadenan actos comunicativos, facilitar los apoyos necesarios para que pueda realizar la actividad y posteriormente retirarlos progresivamente y evitar elementos de distracción.

Es necesario tener en cuenta que solo un 51\% de los participantes en el estudio afirman haber recibido formación específica sobre los trastornos del lenguaje derivados del TEA (Tabla 1.) por lo que puede haber una variación de la efectividad dependiendo de la fracción de la muestra donde se han efectuado la aplicación las diferentes metodologías y estrategias. Igualmente se han aplicado de forma combinada en la totalidad de los casos.

\section{DISCUSIÓN}

En la hipótesis inicial del presente estudio se propone que la detección temprana de los problemas del lenguaje derivados del TEA y su tratamiento se traduce en unos mejores resultados de la intervención educativa. Esta afirmación implica que la detección temprana de dichos trastornos permite obtener evoluciones mas positivas cuanto de aplican de forma inmediata estrategias eficaces para la intervención en el desarrollo del lenguaje. También se proponía recabar información precisa sobre la utilidad de las mencionadas estrategias de intervención, cuáles son las más comúnmente utilizadas por los docentes participantes en el estudio y como han afectado a la evolución de los diferentes casos estudiados. Atendiendo a estos objetivos podemos determinar que el análisis de los datos obtenidos pone de manifiesto la relación es- 
tablecida entre a la edad de detección de los problemas del lenguaje derivados del TEA y su evolución, confirmando la hipótesis de la eficacia de la intervención temprana. Esta formulación revela que en la totalidad de los casos detectados en el rango edad comprendido entre 1-3 años, la evolución del desarrollo educativo en lo que concierne al área del lenguaje fue valorada positivamente, en contraste, en los niños que fueron diagnosticados entre los 4 y los 6 años de edad, la mejoría solo fue evidente en un $56 \%$ de los casos.

Llegar a esta conclusión ha sido posible mediante el diseño cuantitativo del estudio, que ha permitido corroborar la hipótesis planteada, no obstante, una segunda encuesta tomando una muestra mayor durante un periodo de tiempo prolongado podría confirmar los resultados arrojados de forma más concluyente de forma más sólida. Así mismo, como cualquier estudio de estas características, supone unos resultados extrapolables a una población mayor, siempre y cuando se den unas condiciones similares en la población, principalmente acceso a recursos educativos especiales, nivel de desarrollo socio económico de los padres y acceso al sistema educativo entre otras condiciones determinantes.

En favor de los resultados expuestos en el presente trabajo de investigación, los resultados obtenidos en estudios previos, como el realizado en septiembre de 2005 por el programa de detección precoz de trastornos generalizados del desarrollo en las áreas de salud de Salamanca y Zamora, ponen de manifiesto la necesidad de la detección temprana de los problemas derivados del TEA, incluidos los trastornos del lenguaje relacionados, así, un $80 \%$ de los especialistas consultados manifestaron la utilidad de realizar test de manera temprana. Dando fuerza a este enunciado, hasta la fecha no se ha publicado ningún estudio (no se ha encontrado durante la investigación) que muestre resultados opuestos a los expuestos en este artículo.

\section{CONCLUSIONES}

En definitiva, la metodología utilizada y los resultados obtenidos ponen de manifiesto las conclusiones que se presentan a continuación, determinando las implicaciones prácticas de utilidad para el desarrollo de estrategias de intervención en casos de niños con problemas del lenguaje derivados del trastorno autista.

Dados los resultados presentados podemos concluir que la premisa inicial que establece la relación entre la edad de detección y la eficacia de la intervención educativa, demuestra de forma concluyente la importancia de una detección e intervención temprana para la consecución más efectiva del desarrollo del lenguaje en estos casos. 
En este aspecto son relevantes los datos recogidos sobre las metodologías utilizadas en las intervenciones tempranas y la eficacia medida por la apreciación de los especialistas consultados que las han aplicado:

- En cuanto a la relación entre la detección temprana y la mejoría del desarrollo tras aplicar estrategias de intervención se establece como mejor estrategia de detección la Atención especial a la expresión y comprensión de los estados emocionales.

- Una vez diagnosticado el trastorno del lenguaje derivado del TEA, en este orden las estrategias de intervención propuestas con mejores resultados detectados por la muestra de la población docente han sido:

- El establecimiento de rutinas y situaciones muy estructuradas que faciliten la anticipación por parte del alumno.

- Tomar los propios intereses del alumno como punto de partida.

- Preparación de situaciones que favorecen o desencadenan actos comunicativos.

- Facilitar los apoyos necesarios para que pueda realizar la actividad y posteriormente retirarlos progresivamente y evitar elementos de distracción.

Una vez validada la hipótesis de la influencia de la detección temprana, los resultados obtenidos sobre el uso de estrategias de intervención en los casos de problemas del lenguaje derivados del TEA, plantean nuevas preguntas; El estudio no ha determinado, por no ser éste su tema central, si cada estrategia de intervención empleada ha sido más efectiva para cada tipo de trastorno del lenguaje en particular. Esta apreciación abre una nueva línea de investigación dirigida a contrastar los datos obtenidos en este sentido para obtener unos resultados mas precisos.

Otra consideración a tener en cuenta de cara a un posible nuevo estudio es observar que un $51 \%$ de los participantes en el cuestionario afirman haber recibido formación específica sobre los trastornos del lenguaje derivados del TEA, lo que en opinión de la investigación es una cifra insuficiente, pudiendo obtenerse unos datos más precisos para su posterior análisis cribando los resultados en función de la formación del personal docente.

Por último cabe destacar lo que el presente estudio ha aportado a los conocimientos previamente existentes, que ya indicaban la efectividad de la atención temprana en este tipo de casos; Se ha obtenido información de las estrategias empleadas por los profesionales docentes y se ha elaborado un listado con las más relevantes en función de la efectividad registrada en casos reales documentados. 


\section{REFERENCIAS BIBLIOGRÁFICAS}

Altuve, S.(1990). Metodología de la Investigación. Módulo Instruccional. Caracas: Universidad Experimental Simón Rodríguez.

Artigas, J. (1999) Language in autistic disorders. Rev Neurol.

Bisquerra Alzina, R. (coord.) et al.(2004) Metodología de la investigación educativa, Madrid: La Muralla.

Bonilla Catro, E. y Rodríguez Shek, P. Más allá del dilema de los métodos. La investigación en ciencias sociales. $3^{\circ}$ Ed. Santafé de Bogotá, Ediciones uNidades, 1997, Cap. 6. Manejo de datos cualitativos. Pág. 439-478.

Cea D’Ancona, M.A. (1998). Metodología cuantitavia. Estrategias y técnicas de investigación social. Madrid Síntesis.

Cormack, R. M. (1971). The statistical argument. Edinburg: Oliver \& Boyd.

Fortea-Sevilla MS, Escandell- Bermúdez MO, Castro-Sánchez JJ, y Martos-Pérez J. (2015) Desarrollo temprano del lenguaje en niños pequeños con trastorno del espectro autista mediante el uso de sistemas alternativos. Rev Neurol.

Gándara Rossi. Principios y estrategias de intervención educativa en comunicación para personas con autismo: TEACCH (2007) Revista de Logopedia, Foniatría y Audiología, Vol. 27

Hernandez Sampieri, R, Baptista Lucio, P. y Fernandez Collado, C. (2010) Metodología de la investigación. $5^{\circ}$ Ed. México, McGraw-Hill, El análisis de los datos cualitativos.

Lozano Martinez J., Castillo Reche I., García Martínez C., y Motos Guerra E. (2013) The development of social and emotional skills in students with autism spectrum disorder: A collaborative research in pre-school and primary education. EDUCODI Universidad Murcia.

Martos, J y Ayuda, R. (2002) Communication and language in the autistic spectrum: Autism and Dysphasia. Rev Neurol.

Medina y Rodriguez, C. (2016) Potenciar las capacidades de las personas. Modelo para facilitar la comunicación con estudiantes del espectro autista. Revista nacional e internacional de educación inclusiva Vol 9.

Mejía Navarrete, J. (1999) Técnicas cualitativas de investigación en las ciencias sociales. Investigaciones sociales, 3, 22-256.

Rodríguez G., Gil J. y García E. (1996) Metodología de la investigación cualitativa. España, Ediciones Aljibe. 